\title{
Oncogenic Osteomalacia: Diagnosis, Localisation and Cure
}

Elizabeth Mumford BMBCh${ }^{1}$, Joanne Marks $\mathrm{PhD}^{2}$, Thomas Wagner $\mathrm{MBBS}^{3}$, Andrew Gallimore FRCPath ${ }^{4}$, Simon Gane FRCS ${ }^{5}$, Stephen B Walsh FRCP 1

${ }^{1}$ UCL Centre for Nephrology, Royal Free Hospital, University College London.

2Department of Neuroscience, Physiology and Pharmacology, University College London. ${ }^{3}$ Department of Nuclear Medicine, Royal Free Hospital NHS Trust, London. ${ }^{4}$ Department of Pathology, University College London Hospitals, London ${ }^{5}$ Royal National Throat Nose and Ear Hospital, London.

Correspondence to:

Dr Stephen Walsh, UCL Centre for Nephrology, Royal Free Hospital, Rowland Hill Street, London, NW3 2PF

Tel: 02077940500

Stephen.walsh@ucl.ac.uk 
A 37-year-old lady was referred to rheumatology (Treliske Hospital, Truro) in 2015 following 8 months of rib pain, initially thought to be costochondritis. She had isolated hypophosphataemia $(0.50 \mathrm{mmol} / \mathrm{L})$ and a pelvic $\mathrm{MRI}$ identified stress fractures (Figure 1a). 14 months after her presentation she had a persistent low phosphate, with normal PTH, serum calcium and $25(\mathrm{OH})$ vitamin D of $32 \mathrm{ng} / \mathrm{mL}$. It was concluded that "she has subclinical [vitamin D] deficiency" and she was administered cholecalciferol 20,000 units/week and SandoPhos, 2 tablets BD. In March 2016, still symptomatic, she was referred to the UCL Centre for Nephrology to investigate a possible renal cause for the hypophosphataemia.

There, her calculated urinary fractional excretion of phosphate was raised at $42 \%$, confirming hyperphosphaturia. Her gender and age weighed against X-linked, autosomal dominant, or recessive hypophosphtaemic rickets; normal excretion of low molecular weight protein, urate and glucose ruled out the renal Fanconi syndrome. The serum concentration of FGF23 was 180RU/ml (NR 0-150). Previous standard practice protocolled a first-line FDG PET/CT scan, which identified a nasal cavity mass which was avid on a subsequent ${ }^{68} \mathrm{Ga}$ DOTATATE PET/CT (Figure 1b). The tumour was excised and pathology confirmed "Sinonasal mucosa [with] evidence of phosphaturic mesenchymal tumour"; her serum phosphate and FCF23 returned to normal.

Oncogenic osteomalacia is a paraneoplastic syndrome usually associated with mesenchymal tumours, although somatic mutations in adenocarcinomas causing this syndrome have been reported. Phosphatonins (e.g. FGF23) decrease renal resorption of phosphate, leading to hypophosphataemia, muscle weakness and osteomalacia. Most tumours are located in bone and soft tissue, solitary, and rarely cause local symptoms often making the diagnosis long and difficult. Use of 
radiolabelled somatostatin analogues (such as octreotate, as in a ${ }^{68} \mathrm{GA}$-DOTATATE scan) is the most sensitive and specific functional imaging.

The only definitive management is removal of the phosphaturic mesenchymal tumour; recurrence is rare but has been described. 
Figure Legend: Oncogenic Osteomalacia

(A) MRI of sacrum showing stress fractures (indicated by arrow) (B) ${ }^{68} \mathrm{Ga}-$ DOTATATE PET/CT scan showing intense uptake in soft tissue lesion in the left nasal cavity and ethmoid sinus. 


\section{Challenging question}

A 37-year old female presented with bone pain affecting her legs. There was no past medical history and she took no regular medications. A bone scan showed widespread increased uptake consistent with osteomalacia. Her serum phosphate was low at $0.3 \mathrm{mmol} / \mathrm{L}$. Serum calcium, PTH and vitamin D were all normal. Urine analysis showed an increased excretion of phosphate (fractional excretion $45 \%$ ) but normal urinary urate and glucose, with undetectable protein. What is the most likely diagnosis?

(a) X-linked hypophosphatemic rickets

(b) Fanconi syndrome

(c) Oncogenic osteomalacia

(d) Vitamin D deficiency 


\section{Contributors:}

EM: Complied the data and drafted the manuscript

JM: Complied the data and did the biochemical assays

TW: Performed and interpreted the DOTATATE radiology

AG: Interpreted the histopathology

SG: Performed the surgery and supplied the endoscopic surgical images

SBW: Looked after the patient, made the diagnosis and edited the manuscript.

Written informed consent to publication was obtained.

Conflict of Interest Statement:

The authors declared no conflicts of interest. 